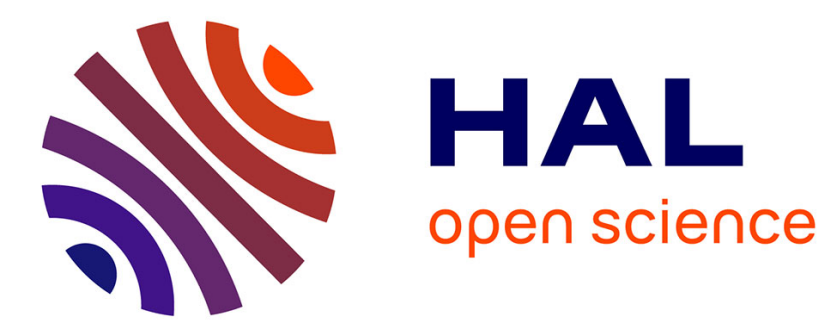

\title{
Dynamical response of dissipative helical edge states
}

Doru Sticlet, Jérôme Cayssol

\section{To cite this version:}

Doru Sticlet, Jérôme Cayssol. Dynamical response of dissipative helical edge states. Physical Review B: Condensed Matter and Materials Physics (1998-2015), 2014, 90 (20), pp.201303 (1-5). 10.1103/PhysRevB.90.201303 . hal-01094367

\section{HAL Id: hal-01094367 https://hal.science/hal-01094367}

Submitted on 12 Dec 2014

HAL is a multi-disciplinary open access archive for the deposit and dissemination of scientific research documents, whether they are published or not. The documents may come from teaching and research institutions in France or abroad, or from public or private research centers.
L'archive ouverte pluridisciplinaire HAL, est destinée au dépôt et à la diffusion de documents scientifiques de niveau recherche, publiés ou non, émanant des établissements d'enseignement et de recherche français ou étrangers, des laboratoires publics ou privés. 


\title{
Dynamical response of dissipative helical edge states
}

\author{
Doru Sticlet* and Jérôme Cayssol ${ }^{\dagger}$ \\ LOMA (UMR-5798), CNRS and University Bordeaux 1, F-33045 Talence, France
}

(Received 26 June 2014; published 4 November 2014)

\begin{abstract}
Quantum spin Hall insulators are characterized by topologically protected counterpropagating edge states. Here we study the dynamical response of these helical edge states under a time-dependent flux biasing, in the presence of a heat bath. It is shown that the relaxation time of the edge carriers can be determined from a measurement of the dissipative response of topological insulator disks. The effects of various perturbations, including Zeeman coupling and disorder, are also discussed.
\end{abstract}

DOI: 10.1103/PhysRevB.90.201303

PACS number(s): 73.63.-b, 73.23.-b, 73.21.Hb

Introduction. The hallmark of two-dimensional (2D) quantum spin Hall (QSH) topological insulators (TIs) consists in the existence of dissipationless conducting edge states in the absence of any time-reversal breaking perturbations [1]. Due to spin-orbit coupling and a particular bulk band structure, the edge carriers' spin is tied to their momentum [2,3]. These helical edge states have been reported experimentally in $\mathrm{HgTe} / \mathrm{CdTe}$ [4] and InAs/GaSb [5] quantum wells. So far, most of the studies have covered the equilibrium or ground-state physics of helical edge states, while less is known about their dynamics and the associated relaxation mechanisms. Only recently, the problem of dissipation has gained attention in the context of topological insulators (TI) [6] and topological superconductors [7].

Recently, it has been proposed that the Floquet type of TIs can be engineered by applying a proper external drive on semimetals or trivial band insulators [8]. Floquet bands have already been reported in time-resolved photoemission experiments on three-dimensional TIs [9], and their topological nature is under active debate. Relaxation phenomena are crucial to establish such nonequilibrium steady states of matter, and ensure the balance between the energy injected by the drive and the energy dissipated towards microscopic degrees of freedom of the environment.

Meanwhile, experimental progress has been achieved in extracting typical relaxation times of carriers in coherent conductors such as normal-superconducting (NS) rings [10,11]. In such experiments, a small coherent system, characterized by a flux-dependent spectrum, is coupled to a multimode superconducting resonator. The dissipative and nondissipative magnetic susceptibility of unconnected samples is obtained by measuring the energy shifts and quality factors of the resonances as a function of frequency, temperature, and dc magnetic flux. In this Rapid Communication, we suggest that these techniques could be applied to extract the typical relaxation times of helical edge carriers circulating around disks of two-dimensional (2D) TIs.

In view of these experimental advances, this Rapid Communication addresses the dynamical response of the generic helical edge state of a 2D TI coupled to a thermal bath and threaded by a time-dependent flux $\Phi(t)$, which is the superposition of a dc flux $\phi$ and a small alternating flux at

\footnotetext{
*doru-cristian.sticlet@u-bordeaux.fr

†jerome.cayssol@u-bordeaux.fr
}

a single frequency $\omega$ (see experiments $[10,11]$ ). It is obtained that the dissipative response of the helical edge state exhibits a characteristic phase-dependent signature: a single peak is located either at $\phi=0$ or at $\phi=\phi_{0} / 2$, depending on the electronic filling. This peak has a maximal amplitude when the frequency is equal to the relaxation rate of the edge carriers. In contrast to standard metallic rings [12,13] or NS rings [10,11], the extraction of the carrier lifetime is simplified by a selection rule which forbids interband transitions between left and right spin-polarized movers. This is a dynamical manifestation of the edge states' helical structure. These analytical results are validated in a comparison with lattice simulations of the Bernevig-Hughes-Zhang (BHZ) model for $\mathrm{HgCd} / \mathrm{CdTe}$ quantum wells [3]. Lastly, this Rapid Communication analyzes the effects of a Zeeman spin-flip coupling and of disorder on the predicted phenomena.

Model and formalism. Let us consider a disk of a 2D TI under a perpendicular time-dependent uniform magnetic field $B(t)$ (Fig. 1). Here, the focus is on the response of the helical edge liquid which encloses the time-dependent magnetic flux $\Phi(t)=\phi+\delta \phi(t), \phi$ being a constant flux and $\delta \phi(t)=\delta \phi_{\omega} \cos \omega t$ being a small oscillating flux. The ac amplitude $\delta \phi_{\omega}$ is kept much smaller than the magnetic flux quantum $\phi_{0}=h / e, h$ being the Planck constant and $e$ the absolute value of the electronic charge. The total Hamiltonian $H$ describing the system decomposes into a static and a dynamic part as $H=H_{0}+H^{\prime}(t)$, with

$$
H_{0}=\frac{h v_{F}}{L}\left(-i \frac{\partial}{\partial \theta}+\frac{\phi}{\phi_{0}}\right) \sigma_{3}, \quad H^{\prime}(t)=\frac{e v_{F}}{L} \delta \phi(t) \sigma_{3} .
$$

The Fermi velocity of the carriers is $v_{F}$, the length of the edge state, $L$, and the angular coordinate, $\theta$. The $\sigma_{3}$ matrix is the standard diagonal spin Pauli matrix.

In the absence of a time-dependent drive $[\delta \phi(t)=0]$, the helical liquid is described by the low-energy effective Hamiltonian $H_{0}$, and it supports a robust persistent current $I_{\text {per }}(\phi)$, characterized by a maximal amplitude $I_{0}=e v_{F} / L$ at zero temperature [14]. The flux-dependent energy levels $\epsilon_{n \sigma}(\phi)=\epsilon_{\mathbf{n}}(\phi)=\sigma \hbar \omega_{0}\left(n+\phi / \phi_{0}\right)$ are discrete and identified by an angular momentum $n$ and a spin $\sigma$ quantum numbers, which are gathered in the notation $\mathbf{n}=(n, \sigma)$. The corresponding energy eigenstates solve the Schrödinger equation $H_{0}|\mathbf{n}\rangle=\epsilon_{\mathbf{n}}(\phi)|\mathbf{n}\rangle$, where $|\mathbf{n}\rangle$ are the eigenspinors of $\sigma_{3}$ times $e^{i n \theta}$. The energy spacing between adjacent levels of a given 


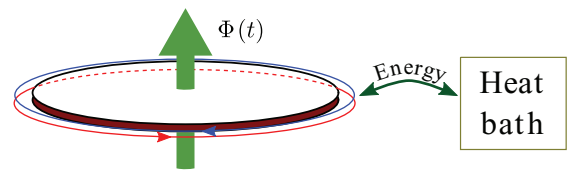

FIG. 1. (Color online) A QSH TI disk under a time-dependent perpendicular magnetic field $B(t)$. The Hamiltonian (1) models the counterpropagating edge states (red and blue) which enclose a flux $\Phi(t)=\phi+\delta \phi(t)$. The dc flux $\phi$ is varied arbitrarily, while the timedependent oscillatory flux $\delta \phi(t)=\delta \phi_{\omega} \cos \omega t$ has a small amplitude with respect to the flux quantum. The edge carriers are exchanging energy with a heat bath and the dynamical susceptibility $\chi(\omega)$ gains a dissipative component at finite frequency.

spin and flux is denoted by $\hbar \omega_{0}=h v_{F} / L$. Each energy level carries a flux-independent current $i_{\mathbf{n}}=-\sigma I_{0}=-\sigma e v_{F} / L$.

Let us consider that the quantum edge states are coupled to a thermal bath containing many degrees of freedom. These degrees of freedom could have various distinct microscopic origins: electromagnetic modes of the external circuit, phonons, bulk states of the disk, etc. Then, in response to the finite driving term $\delta \phi(t)=\delta \phi_{\omega} \cos \omega t$, the edge supports both nondissipative $\left(I_{\mathrm{ac}}^{\prime} \cos \omega t\right)$ and dissipative $\left(I_{\mathrm{ac}}^{\prime \prime} \sin \omega t\right)$ ac steady currents. This response is captured by a complex frequencydependent susceptibility $\chi(\omega)=\chi^{\prime}(\omega)+i \chi^{\prime \prime}(\omega)$, defined by $\chi^{\prime}(\omega)=I_{\omega}^{\prime} / \delta \phi_{\omega}$ and $\chi^{\prime \prime}(\omega)=I_{\text {ac }}^{\prime \prime} / \delta \phi_{\omega}$. In the present setup, the TI disk is unconnected and therefore it only exchanges energy with the environment, while the number of particles remains fixed.

Here, we will not investigate the microscopic mechanisms leading to dissipation, but rather provide a generic and phenomenological model to describe it in the case of a weak coupling to the environment. To this aim, we consider the evolution of the system under the following kinetic equation for the reduced (single-particle) density operator $\rho(t)$ (obtained after tracing out the environment degrees of freedom $[12,13,15])$ :

$$
\frac{\partial \rho(t)}{\partial t}+\frac{i}{\hbar}[H(t), \rho(t)]=-\gamma\left[\rho(t)-\rho_{\mathrm{qe}}(t)\right],
$$

where $\quad \rho_{\mathrm{qe}}(t)=\left\{\exp \left[(H(t)-\mu) / k_{B} T\right]+1\right\}^{-1} \quad$ is the quasiequilibrium density matrix at temperature $T, k_{B}$ being the Boltzmann constant. The matrix $\gamma$ phenomenologically represents the relaxation rates for populations and coherences in the density matrix operator. Because the system exchanges only heat with the environment, the number of particles is fixed. Consequently, the chemical potential $\mu$ is not constant and generally depends on flux, number of particles, temperature, and time. Nevertheless, $\mu$ can be taken here constant in flux, due to the particular flux dependence of the last occupied energy level for a given parity of electron number. Moreover, the time dependence of $\mu$ brings only a negligible contribution to the dissipative response in comparison with other competing terms [16].

In the linear response approximation $\left(\delta \phi_{\omega} \ll \phi_{0}\right)$, the master equation (2) is solvable, and the complex linear susceptibility can be decomposed into three parts [12,13],

$$
\chi(\omega, \phi)=\chi_{\mathrm{per}}+\chi_{D}(\omega, \phi)+\chi_{N D}(\omega, \phi) .
$$

The static part of the susceptibility $\chi_{\text {per }}$ is purely real and it is due to the persistent current in the system. The second and third terms are called diagonal and nondiagonal with reference to the $H_{0}$ eigenstate basis. The diagonal susceptibility $\chi_{D}$ describes only the intraband response of the system, while the nondiagonal susceptibility $\chi_{N D}$ is related to interband transitions. Note that all the terms in Eq. (3) depend also on temperature.

Helical edge states' susceptibility. The static part of the susceptibility $\chi_{\text {per }}=\frac{\partial}{\partial \phi}\left(\sum_{\mathbf{n}} i_{\mathbf{n}} f_{\mathbf{n}}\right)$ is the derivative of the persistent current with respect to the dc flux $\phi$. In this case, the sum runs over the angular momentum and spin quantum numbers. The functions $f_{\mathbf{n}}$ represent henceforth the Fermi-Dirac distribution function for static Hamiltonian $H_{0}$, $f_{\mathbf{n}}=f\left(\epsilon_{\mathbf{n}}(\phi)\right)$.

The perturbation $H^{\prime}(t)$ commutes with $H_{0}$ and it cannot induce spin flips or changes in the angular momentum of the electrons. Because the system does not exchange electrons with the environment the spin and angular quantum numbers remain conserved. This selection rule forbids interband transitions and it implies that the nondiagonal susceptibility $\chi_{N D}(\omega, T, \phi)$ vanishes. Therefore, dissipation can occur only through intraband relaxation processes.

This is a remarkable simplification with respect to the case of multilevel systems encountered in experiments for normal (and Josephson) rings, where separating the three contributions in Eq. (3) is a difficult and subtle task [11,13,17]. Therefore, the linear susceptibility of the helical edge contains only two terms: $\chi(\omega, \phi)=\chi_{\text {per }}+\chi_{D}(\omega)$. Furthermore, the dissipative part of the susceptibility has only one term, $\chi^{\prime \prime}(\omega)=\chi_{D}^{\prime \prime}(\omega)$, since $\chi_{\text {per }}$ is purely real (nondissipative). Moreover, the diagonal rates $\gamma_{\mathbf{n n}}$ are assumed to be all identical $\gamma_{\mathbf{n n}}=\gamma_{D}$ and flux independent, since the energy levels are equidistant and have the same absolute value of the level current. The dissipative response $\chi_{D}^{\prime \prime}$ is given by the imaginary part of the diagonal susceptibility [16],

$$
\chi_{D}(\omega, \phi)=\frac{\gamma_{D}}{i \omega-\gamma_{D}} \sum_{\mathbf{n}} i_{\mathbf{n}}^{2} \frac{\partial f_{\mathbf{n}}}{\partial \epsilon_{\mathbf{n}}},
$$

and it is maximal for $\omega=\gamma_{D}$ (Fig. 2). The edge states' lifetime $\gamma_{D}^{-1}$ can then be measured from dissipative response by sweeping the driving frequency.

The explicit result for dissipative susceptibility as a function of temperature, flux, frequency, and chemical potential $\mu$ reads as [16]

$$
\begin{aligned}
\frac{\chi_{D}^{\prime \prime}(\omega, \phi)}{\chi_{0}}= & \frac{4 \omega \gamma_{D}}{\omega^{2}+\gamma_{D}^{2}}\left[\frac{1}{2}+\sum_{m=1}^{\infty} \frac{m T / T^{*}}{\sinh \left(m T / T^{*}\right)}\right. \\
& \left.\times \cos \left(2 \pi m \frac{\phi}{\phi_{0}}\right) \cos \left(2 \pi m \frac{\mu}{\hbar \omega_{0}}\right)\right],
\end{aligned}
$$

in units of $\chi_{0}=\frac{I_{0}}{\phi_{0}}=\frac{e^{2} v_{F}}{h L}$. The characteristic temperature $T^{*}$ is proportional to the level spacing, $T^{*}=\hbar v_{F} /\left(\pi k_{B} L\right)$. It immediately follows that the dissipative susceptibility of the current is peaked at zero flux (Fig. 2). If the fermionic parity is changed by adding or subtracting a single particle, the chemical potential changes by $\hbar \omega_{0} / 2$ and the peak moves to half-integer flux $\phi / \phi_{0}= \pm 0.5$ [16]. 

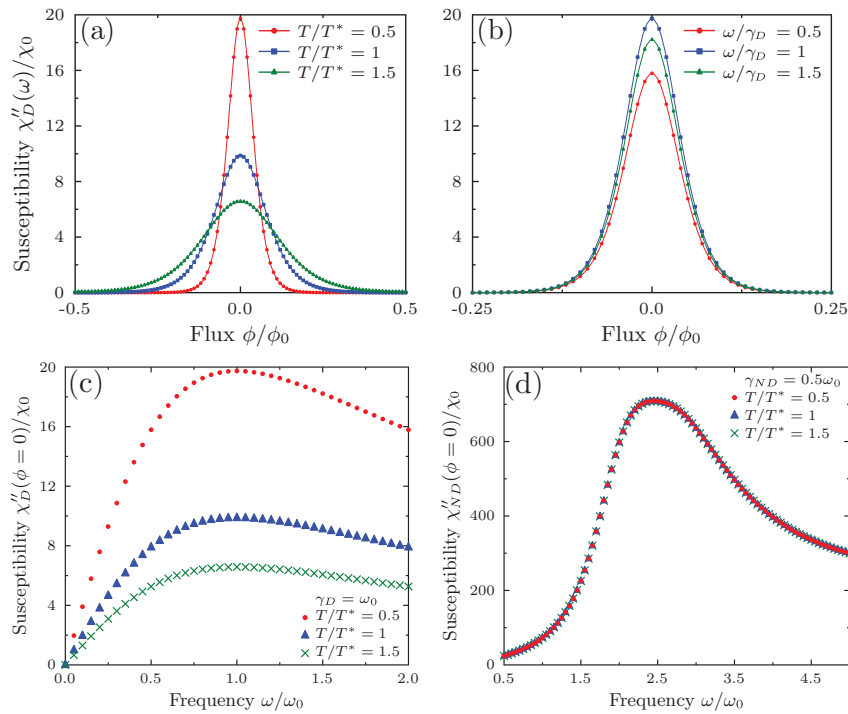

FIG. 2. (Color online) For the clean helical edge, the dissipative susceptibility $\chi_{D}^{\prime \prime}(\phi, \omega)$ has a peak at zero flux $\phi=0$ (a) and is maximal at frequency $\omega=\gamma_{D}^{-1}[(\mathrm{~b})$ and (c)]. The analytical result (continuous lines) and the Bernevig-Hughes-Zhang (BHZ) lattice model (markers) coincide (after multiplying by a factor 2 the single helical edge result, in order to take into account the presence of two edges in the lattice simulations). (d) In the lattice model, there is a large nondiagonal contribution $\chi_{N D}^{\prime \prime}$ which is independent of the temperature. Parameters of the system (unless otherwise stated): temperature $T=0.5 T^{*}$, frequency $\omega=\omega_{0}$, and lattice size $\left(L_{x}, L_{y}\right)=(80,80) a$. In the BHZ model $(A, B, M)=(1,0.6,1)$.

Since the current matrix is diagonal, there is no damping rate for coherences $\rho_{\mathbf{m n}}(\mathbf{m} \neq \mathbf{n})$, and the dissipation is entirely captured by the evolution for the populations $\rho_{\mathbf{n n}}$ as in [18]. The eventual contribution of bulk states in the insulating disk will be discussed below in connection with the lattice simulations.

Comparison with the BHZ model. We now present numerical simulations supporting the analytical results above. We use the Bernevig-Hughes-Zhang (BHZ) model on a square lattice, described by the Hamiltonian [3]

$$
\begin{aligned}
H= & \sum_{x=1}^{L_{x}} \sum_{y=1}^{L_{y}-1} c_{x y}^{\dagger}\left[\left(\frac{A}{2 i} \sigma_{1} \tau_{3}+B \sigma_{3} \tau_{0}\right) e^{i \frac{\varphi(t)}{L_{x}}} c_{x+1 y}\right. \\
& \left.+\left(\frac{A}{2 i} \sigma_{2}+B \sigma_{3}\right) \tau_{0} c_{x y+1}+\left(\frac{M}{2}-2 B\right) \sigma_{3} \tau_{0} c_{x y}\right] \\
& + \text { H.c., }
\end{aligned}
$$

$A, B$, and $M$ being material parameters, and $\varphi(t)=$ $2 \pi \Phi(t) / \phi_{0}$ the time-dependent phase induced by the applied flux (lattice constant $a=1$ ). This model is a useful lattice regularization of the effective 4-band Dirac model describing the topological transition in $\mathrm{HgTe} / \mathrm{CdTe}$ quantum wells [3]. The different terms are tensor products of the Pauli matrices $\sigma$ and $\tau$ describing internal degrees of freedom. We use the hollow cylinder geometry, with base circumference $L_{x}$ and height $L_{y}$. The system is taken in a topological insulating phase (bulk gap $\simeq 2 A$ ) and at half filling $N_{1 / 2}=2 L_{x} L_{y}$. Then the model in Eq. (6) exhibits a pair of counterpropagating helical edge states located at the bottom $(y=1)$ [and one at the top $\left(y=L_{y}\right)$ ] base of the cylinder. At low energy, each pair of edge states is described by the helical model in Eq. (1) with $v_{F}=a A / \hbar$. The mapping between the two models requires that the temperature $k_{B} T$ is taken much smaller than the BHZ bulk gap and also that $L_{y}$ is large enough to avoid overlap between these two edge states.

In Figs. 2(a) and 2(b) the diagonal susceptibility shows a peak at zero flux which is maximal when the frequency is exactly equal to the relaxation rate $\gamma_{D}$. The magnetic signal from the helical model [Eq. (1)] is scaled by a factor of 2 in order to account for the two helical edge liquids in the lattice BHZ model (top and bottom of the cylinder). The match between the helical and the BHZ models holds at different driving frequencies, temperatures, or diagonal rates $\gamma_{D}$. Indeed, the diagonal susceptibility depends crucially on the states near the chemical potential and thus at half filling it is well approximated by that of the edge states inhabiting the gap, while the bulk contribution is negligible. The two pairs of edge states must be well separated otherwise hybridization of edge states leads to a vanishing zero-flux susceptibility. Furthermore, the diagonal susceptibility in zero flux decreases with temperature, but it maintains a maximum at $\omega=\gamma_{D}$ [Fig. 2(c)]. If a pair of particles is added, the susceptibility-flux characteristic is shifted by half-integer flux quantum, such that the susceptibility peak moves to $\phi / \phi_{0}=0.5$. At odd number of particles the peaks are smaller and appear at both $\phi / \phi_{0}=0$ and 0.5 [16].

In contrast with the $1 \mathrm{D}$ helical model Eq. (1), the 2D lattice model allows transitions between the bulk states. These transitions induce a large contribution only to the nondiagonal susceptibility $\chi_{N D}^{\prime \prime}(\omega) \propto L_{x} L_{y}$ [Fig. 2(d)], which scales with the number of electrons in the system, while the diagonal contribution scales with the edge length, $\chi_{D}^{\prime \prime}(\omega) \propto$ $L_{x}$. Nevertheless, this large bulk-state contribution is almost flux independent in the thermodynamic limit, thereby allowing an easy extraction of the flux-dependent edge contribution [16] and determination of the lifetime $\gamma_{D}^{-1}$ of the edge states. Note that the nondiagonal dissipative response $\chi_{N D}^{\prime \prime}$ has been evaluated under the assumption that all damping rates for coherences [in Eq. (2)] are equal and constant in flux or temperature, $\gamma_{\mathbf{m n}}=\gamma_{N D}$.

Disorder effects. The addition of scalar disorder does not destroy the edge states. The signature peak in the dissipative diagonal susceptibility slowly decreases; however it does not vanish, if the disorder strength is smaller than the bulk gap $[\simeq 2 A$ for the parameters in simulations of Fig. 3(d)]. As disorder strength increases and becomes larger than the bulk gap, dips can develop in the diagonal susceptibility in random samples. On average, the susceptibility at large disorder becomes more and more flat and flux independent [Fig. 3(d)].

It is important to remark that this situation is different from the case of a regular system with nonrelativistic fermions. Indeed, rings with nonrelativistic fermions present energy level crossings in the ballistic limit which are not protected against disorder: infinitesimal scalar disorder removes the degeneracies and yields a vanishing zero-flux diagonal susceptibility (instead of the peak predicted in the topologically protected edge state).

Effect of an in-plane field. An additionally static field induces a Zeeman coupling between spin up and spin down. 

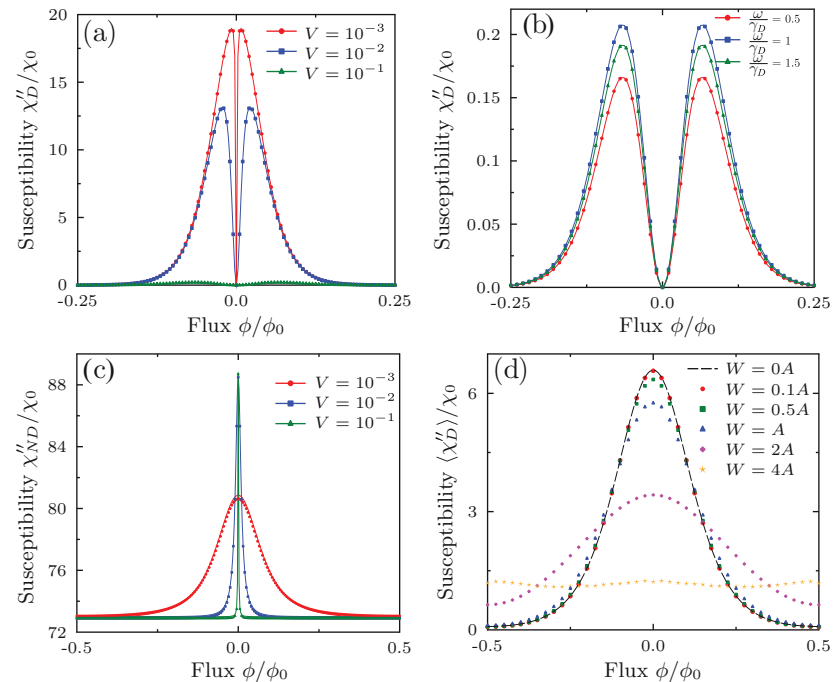

FIG. 3. (Color online) The dissipative susceptibility under the effect a small uniform Zeeman field (a), (b), and (c), or under scalar disorder with strength $W(\mathrm{~d})$. Both helical model (continuous lines) and BHZ (markers) develop a nondiagonal susceptibility. (a), (b) The diagonal part of the dissipative susceptibility vanishes in zero flux even for a small perturbation $V$. (c) The dissipative nondiagonal susceptibility $\chi_{N D}^{\prime \prime}$ contains a large flux-independent contribution from BHZ bulk states. The response in the gapped helical model, translated by a constant value, matches the lattice result. The usual Zeeman energy is $V=0.1 \hbar \omega_{0}$. Lattice size $\left(L_{x}, L_{y}\right)=(80,80) a$ and temperature $T=0.5 T^{*}$. (d) The average diagonal susceptibility $\left\langle\chi_{D}^{\prime \prime}\right\rangle$ over 200 disorder realizations. The temperature is $T=1.5 T^{*}$ and lattice size $\left(L_{x}, L_{y}\right)=(8,60) a$. The BHZ parameters are $(A, B, M)=$ $(1,0.6 .1)$, and the diagonal response is maximal for $\omega / \gamma_{D}=1$.

In the helical model, we consider a constant term proportional to a spin-mixing matrix $\sigma_{1}, H=H_{0}+H^{\prime}(t)+V \sigma_{1}$. In this case the edge states are gapped out in zero flux, leading to a vanishing dissipative diagonal susceptibility. Moreover, the in-gap states now bring a nondiagonal susceptibility $\chi_{N D}[16]$. The response will depend on the nondiagonal damping rates $\gamma_{\mathbf{m n}}$, which renders the analysis more difficult.

The agreement between the helical and BHZ models still holds [Figs. 3(a) and 3(b)]. The bulk is largely unaffected by the flux, and its contribution to susceptibility remains almost constant in flux. The features in the nondiagonal susceptibility can be accounted for by the edge state contribution, shifted with a large constant dissipative bulk contribution [Fig. 3(c)]. Note that very small spin mixing still opens a gap at timereversal invariant fluxes. Then the vanishing level current leads to a dip in diagonal susceptibility at zero flux. Only energy states close to these flux values are affected for very small Zeeman fields. These lead to dips in the diagonal susceptibility of small width in comparison to the overall width of the signal.

Using the material parameters [4] of the $\mathrm{HgTe} / \mathrm{CdTe}$ quantum wells, we estimate the relevant quantities. The Fermi velocity for $\mathrm{HgTe} / \mathrm{CdTe}$ quantum wells of thickness $d \simeq 7 \mathrm{~nm}$ is approximately $v_{F} \simeq 5.5 \times 10^{5} \mathrm{~m} / \mathrm{s}$. Therefore the characteristic temperature for a ring of size $L=0.5 \mu \mathrm{m}$ is $T^{*} \simeq 2.7 \mathrm{~K}$. The distance between levels at the Fermi surface is $\hbar \omega_{0}$, which for our given wire sets the characteristic frequency $\omega_{0} \simeq 6.9 \times 10^{12} \mathrm{~s}^{-1}$. The characteristic current for the same ring length $I_{0} \simeq 176 \mathrm{nA}$. Therefore the characteristic dimensional susceptibility reads $\chi_{0} \simeq 4.26 \times 10^{7} \mathrm{H}^{-1}$. In order to explore the physics of the edge states, the temperature was taken smaller than the gap [where the gap $\simeq 2$ for $(A, B, M)=$ $(1,0.6,1)]$. Finally we note that the kinetic equation approach is valid only for temperatures larger than the level broadening, which is the case in all the simulations.

Conclusions. In this Rapid Communication, we have studied the dissipative response of a 2D QSH insulator under the effect of a small time-dependent driving in flux. Using a helical model for the edge states and exact diagonalization of a tightbinding $\mathrm{BHZ}$ insulator, we have proven that the contribution of the edge states and the bulk can be differentiated. Crucially, the lifetime of the edge states can be identified by measuring the frequency where the dissipative response is maximal. While the bulk may bring a large contribution to susceptibility, it can be eliminated by observing that it is almost constant in flux.

Moreover, the diagonal dissipative susceptibility is sensitive to the gapping of the edge states (either due to hybridization between pairs of edge states brought in spatial proximity or due to a Zeeman fields at zero flux). The peak in the diagonal susceptibility may split into two (or even more) peaks in these cases.

Acknowledgments. The authors thank H. Bouchiat and B. Dassonneville for introducing them to the topic of dissipation measurements in mesoscopic rings and are grateful to $\mathrm{R}$. Avriller for careful reading of the manuscript. D.S. also thanks F. Piéchon and J.-N. Fuchs for stimulating discussions. This work was supported by the French ANR through projects ISOTOP and MASH.
[1] M. Z. Hasan and C. L. Kane, Rev. Mod. Phys. 82, 3045 (2010); X.-L. Qi and S.-C. Zhang, ibid. 83, 1057 (2011).

[2] C. L. Kane and E. J. Mele, Phys. Rev. Lett. 95, 226801 (2005).

[3] B. A. Bernevig, T. L. Hughes, and S.-C. Zhang, Science 314, 1757 (2006).

[4] M. König, S. Wiedmann, C. Brüne, A. Roth, H. Buhmann, L. W. Molenkamp, X.-L. Qi, and S.-C. Zhang, Science 318, 766 (2007); A. Roth, C. Brüne, H. Buhmann, L. W. Molenkamp, J. Maciejko, X.-L. Qi, and S.-C. Zhang, ibid. 325, 294 (2009).
[5] I. Knez, R.-R. Du, and G. Sullivan, Phys. Rev. Lett. 107, 136603 (2011).

[6] O. Viyuela, A. Rivas, and M. A. Martin-Delgado, Phys. Rev. B 86, 155140 (2012); A. Rivas, O. Viyuela, and M. A. MartinDelgado, ibid. 88, 155141 (2013).

[7] S. Diehl, E. Rico, M. A. Baranov, and P. Zoller, Nat. Phys. 7, 971 (2011); C.-E. Bardyn, M. A. Baranov, C. V. Kraus, E. Rico, A. İmamoğlu, P. Zoller, and S. Diehl, New J. Phys. 15, 085001 (2013).

[8] T. Oka and H. Aoki, Phys. Rev. B 79, 081406 (2009); N. H. Lindner, G. Refael, and V. Galitski, Nat. Phys. 7, 490 (2011); 
T. Kitagawa, T. Oka, A. Brataas, L. Fu, and E. Demler, Phys. Rev. B 84, 235108 (2011); J. Cayssol, B. Dòra, F. Simon, and R. Moessner, Phys. Status Solidi (RRL) 7, 101 (2013); M. S. Rudner, N. H. Lindner, E. Berg, and M. Levin, Phys. Rev. X 3, 031005 (2013).

[9] Y. H. Wang, H. Steinberg, P. Jarillo-Herrero, and N. Gedik, Science 342, 453 (2013).

[10] F. Chiodi, M. Ferrier, K. Tikhonov, P. Virtanen, T. T. Heikkila, M. Feigelman, S. Gueron, and H. Bouchiat, Sci. Rep. 1, 3 (2011).

[11] B. Dassonneville, M. Ferrier, S. Guéron, and H. Bouchiat, Phys. Rev. Lett. 110, 217001 (2013).

[12] N. Trivedi and D. A. Browne, Phys. Rev. B 38, 9581 (1988).

[13] B. Reulet and H. Bouchiat, Phys. Rev. B 50, 2259 (1994).

[14] D. Sticlet, B. Dóra, and J. Cayssol, Phys. Rev. B 88, 205401 (2013).
[15] A. Larkin and Y. N. Ovchinnikov, Sov. Phys. JETP 64, 185 (1986); D. A. Browne, K. S. Chow, and V. Ambegaokar, Phys. Rev. B 35, 7105 (1987); U. Weiss, Quantum Dissipative Systems, 3rd ed., Series in Modern Condensed Matter Physics (World Scientific Publishing Company, Singapore, 2008).

[16] See Supplemental Material at http://link.aps.org/supplemental/ 10.1103/PhysRevB.90.201303 for the review of linear response theory in mesoscopic rings and its application in the derivation of the analytical and numerical results in the article. The dependence of susceptibility on the number of particles away from half filling is also discussed.

[17] M. Ferrier, B. Dassonneville, S. Guéron, and H. Bouchiat, Phys. Rev. B 88, 174505 (2013).

[18] R. Landauer and M. Büttiker, Phys. Rev. Lett. 54, 2049 (1985). M. Büttikker, Ann. N. Y. Acad. Sci. 480, 194 (1986). 